\title{
Lipids
}

Makrides M, Ochoa JB, Szajewska H (eds): The Importance of Immunonutrition. Nestlé Nutr Inst Workshop Ser, vol 77, pp 145-154, (DOI: 10.1159/000351397) Nestec Ltd., Vevey/S. Karger AG., Basel, @ 2013

\section{Clinical Overview of Effects of Dietary Long-Chain Polyunsaturated Fatty Acids during the Perinatal Period}

\author{
Susan A. Scholtz · John Colombo · Susan E. Carlson \\ University of Kansas Medical Center, Kansas City, KS, and University of Kansas, Lawrence, KS, USA
}

\begin{abstract}
The current report provides a brief background introducing 30 years of research on longchain polyunsaturated fatty acids (LC-PUFA) and infant development, but focuses mainly on challenges for future studies. Infants fed formulas containing only vegetable fats were found to have lower docosahexaenoic acid (DHA, 22:6n-3) and arachidonic acid (20:4n-6) status than infants fed human milk. Studies soon focused on efforts to improve LC-PUFA status and evaluate functions suggested by early primate studies of DHA deficiency. Despite evidence for the importance of these fatty acids for development, particularly DHA, several recent meta-analyses conclude dietary supplementation does not enhance development. Future studies should employ (1) more finely grained measures of brain development as opposed to global measures, and (2) tests that evaluate development later in childhood when children are able to be tested on more complex behaviors (if found effective these would also be evidence of early brain programming). (3) Studies are needed to understand the cause of high variability in transfer of DHA to the fetus. (4) Finally, the role of single-nucleotide polymorphisms of the fatty acid desaturase genes 1 and 2 of mother and infant needs study to determine how they affect requirements for these fatty acids by the fetus/infant.

Copyright $\odot 2013$ Nestec Ltd., Vevey/S. Karger AG, Basel
\end{abstract}

\section{Introduction}

Two long-chain polyunsaturated fatty acids (LC-PUFA) that have been studied in relation to infant and child development are considered: docosahexaenoic acid (DHA) and arachidonic acid (ARA), respectively, members of the n-3 or $\omega 3$ 


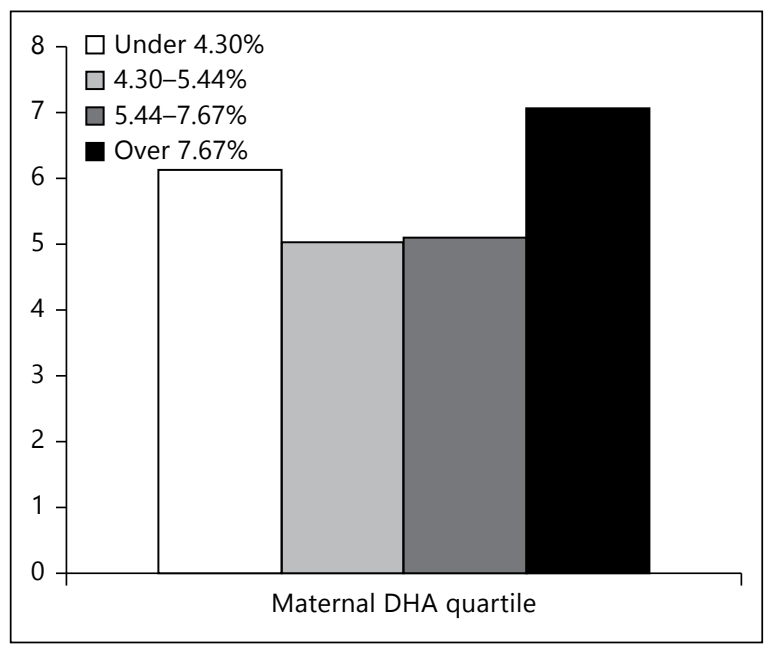

Fig. 1. Shorter look duration is more advanced at 4 months of age. Maternal RBC DHA quartile ANCOVA, controlling for postnatal breastfeeding, maternal education, and infant gender. Maternal DHA quartile effect: $F(3,68)=2.96, p=0.038$, partial $\eta^{2}=0.115$, quadratic contrast $p=0.027$.

and n-6 or $\omega 6$ fatty acid families, and synthesized from linoleic and $\alpha$-linolenic acids (see fig. 1). These LC-PUFA are found preformed in fat of animal sources, including human milk. US infants fed human milk instead of one of the infant formulas available in the late 1970s (which did not contain LC-PUFA) were observed to have a greater proportion of DHA and ARA in their red blood cell membrane phospholipids [1]. Studies by Clandinin et al. [2, 3] had just demonstrated the rapid accumulation of DHA that occurred in the fetal and newborn brain beginning at around 24 weeks gestation and continuing after birth. These studies also suggested that there was considerable variability among infants of the same gestational age in brain DHA accumulation. Later studies of brain composition in human milk compared to formula-fed term and preterm infants suggested postnatal DHA accumulation was increased by DHA intake $[4,5]$. The first outcome measures chosen for study were visual acuity and early cognitive development [6-8]. Martha Neuringer and her colleagues found reduced retinal and brain DHA and lower visual acuity and less mature attention in rhesus monkeys fed diets low in a-linolenic acid during development $[9,10]$. Her studies provided clear evidence that retinal and brain DHA were important for optimal visual acuity and a measure of early learning in n-3 deficiency. In contrast to the nonhuman primate studies, however, infants fed formula supplemented with DHA (with a couple of exceptions) were compared with infants fed formula containing a-linolenic acid. Thus, the human studies were primarily 
designed to ask if biochemical pathways for synthesis of DHA and ARA from their essential 18 carbon precursor provided optimal DHA and ARA for function of infants, i.e. if DHA and ARA were conditionally essential nutrients for the developing infant.

The first postnatal supplementation studies were done in the US in preterm infants, and supplementation was based on the amount of DHA in breast milk of US women $(\sim 0.2 \%$ of total fatty acids). Preterm infants fed formulas with DHA uniformly demonstrated higher (but not necessarily optimal) visual acuity with $0.2 \%$ DHA compared to infants fed formulas without DHA [11]. Later, milk DHA content in the US was demonstrated to be among the lowest in the world. Both term and preterm infants have now been fed up to $1 \%$ of fatty acids as DHA. Preterm infants in the DINO trial did not benefit from $1 \% \mathrm{n}-3 \mathrm{LC}$ PUFA provided until expected term age, but the control group received $\sim 0.3 \%$ n-3 LC-PUFA [12]. In the only dose-response study in term infants that included a $0 \%$ LC-PUFA control, groups of US infants fed DHA $(0.32,0.64$ or $0.96 \%$ of total fatty acids and $0.64 \%$ ARA) had similar visual acuity at one year of age, but their visual acuity was higher than that of infants not fed LC-PUFA [13].

The cognitive assessment of preterm and term infants has been more controversial with recent large trials in preterm infants supplemented after birth [12] or term infants supplemented during gestation (DOMInO trial) [14] reaching the conclusion that supplementation with DHA (or higher DHA) did not result in higher cognitive outcomes at 18 months of age. At least 4 recent meta-analyses or systematic reviews [commented in Colombo and Carlson 15], have reached a similar conclusion. While we do not disagree with the findings of these reviews, as will be explained, neither do we believe that they are clear evidence for a lack of benefit as we have written in a recent commentary [15]. The reasons will be addressed below using data (mainly from our own recent studies). Challenges for new studies will also be addressed. Another chapter presents the effects of LC-PUFA on immune development. LC-PUFA are important constituents of all cell membranes, and DHA intake increases the DHA content in all tissues studied to date. Consequently, DHA supplementation may influence areas of development other than neural and immune development.

\section{Choice of Developmental Measures}

Unlike visual acuity, which was chosen as an outcome because of the work of Neuringer and others, the initial choice of developmental measures in LC-PUFA supplementation studies was not based on theoretically based expectations of where an effect of LC-PUFA might be expected to be seen on development. The 
majority of studies have relied upon global assessments of development, most often the Bayley Scales of Infant Development (BSID). Two subscales of the BSID have been commonly measured in LC-PUFA trials: the Mental Developmental Index (MDI) and the Psychomotor Developmental Index. The BSID (now in its 3 rd version) is normed to a score of 100 and was developed as part of a strategy used in the 1930s through the 1950s to characterize infant/early child development (including developmental delays) in terms of comparison to the normative or average age at which particular psychomotor developmental milestones (such as sitting up, crawling, walking, talking, imitating, and others) were attained. The BSID MDI scores from the first year have been repeatedly shown to be largely unrelated to any later measures of cognition or achievement in later life, while scores during the 2 nd year (e.g. at 18 months) are only modestly related to school-age cognitive performance [16]. Of course, indices of later cognition and achievement are the outcomes that LC-PUFA researchers would most like to assess and influence by supplementation. The BSID continues to be used because pediatricians are familiar with the test, and because results from different studies can easily be compared. Because the BSID is the most common outcome measured in LC-PUFA trials, the systematic reviews or metaanalyses that have evaluated cognitive development rely heavily on results of the BSID MDI [15]. As noted above, four recent reviews have concluded there are no benefits of LC-PUFA for development based largely on results from the BSID.

Meanwhile, there has been evidence for some time that DHA status affects more finely grained measures of cognition in infancy and early childhood, such as attention, memory, processing speed, and other indices of higher order intellectual function. These studies also look to work of Neuringer and her coworkers [10], who linked brain DHA accumulation to attention and information processing. Such tests or assays are typically ignored in reviews, because they are not normalized or standardized; their use in clinical studies requires interdisciplinary collaboration between pediatric/nutrition trialists and developmental cognitive neuroscientists. There have been attempts to harmonize among centers. Generally speaking, many of these early and more granular measures of cognition are associated at modest but statistically significant levels with intelligence later in childhood [17].

We have published a positive correlation between maternal DHA status at delivery and attention/processing speed in infancy and lower distractibility in toddlerhood $[18,19]$ and increased sustained attention in infants fed DHA and ARA for the first year of life (DIAMOND) [20]. In a cohort from another recently completed supplementation trial (KUDOS) that is not yet published, the lowest and highest quartiles of maternal DHA status at delivery had longer du- 


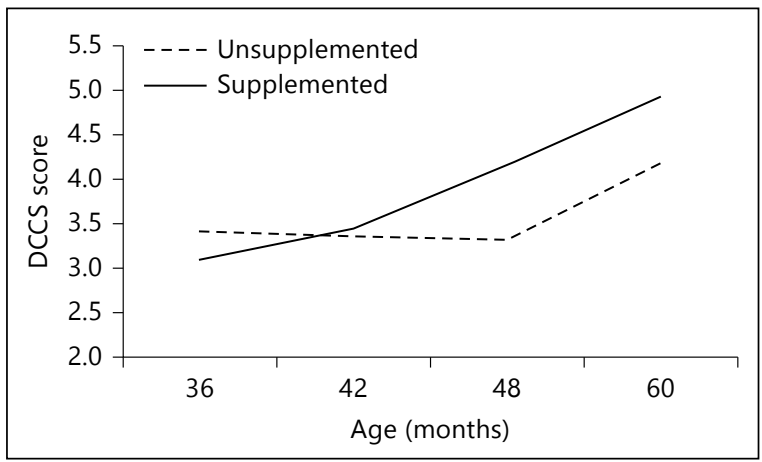

Fig. 2. Dimensional change card sort (DCCS) at each age by LC-PUFA supplementation. Visit, $\mathrm{p}=0.000$; supplemented, $\mathrm{p}=0.071$; visit $\times$ supplemented, $\mathrm{p}=0.006$.

ration looking compared to the middle quartiles (quartile effect $\mathrm{p}<0.038$; fig. 1). Thus, we have positive evidence of an effect of LC-PUFA (particularly DHA status) on early development from 3 separate clinical studies: an observational study, a clinical trial of LC-PUFA supplementation for the first 12 postnatal months, and a clinical trial that provided DHA during almost 2 trimesters of gestation.

\section{Timing of Measurement of Development}

We have already noted in an earlier publication, that 'higher order cognitive functions reach full maturity at ages well beyond the ages to which most RCTs have extended', and that success on tests of 'inhibitory control, rule and strategy use and working memory' develops between 3 and 6 years of age [21]. Trials conducted by Helland et al. [22] and by Jensen et al. [23] provided a source of maternal DHA, respectively, during pregnancy and lactation or lactation alone. As described in Colombo et al. [20], the children of supplemented women in these respective trials had evidence of higher cognition at 4 and 5 years of age even though benefit was not detected in infancy.

In the dose-response trial referred to above (DIAMOND), cognitive development of children was tested at 6-month intervals between 18 months and 6 years of age. Those who did not receive formula with LC-PUFA as infants had poorer performance on tests of inhibitory control and on rule and strategy use. Figure 2 illustrates the effect of age and supplementation on performance on the dimensional card sort task, which tests ability to respond to rule changes (a cognitive function). In this case, the child was asked to sort the cards in one dimension 
(color) and then change to sorting the cards in another dimension (object pictured on the card) [24]. There was an effect of age and also an interaction between age and supplementation. Results of other tests between 18 months and 6 years will be shared.

Adding our DIAMOND results to those of Helland and Jensen and their coworkers mentioned above, there are now 3 randomized clinical studies that show benefits of LC-PUFA supplementation during gestation and/or infancy on cognitive function near school age. There are also numerous observational studies that link higher DHA exposure early in development to higher performance at older ages as will be described. Both the randomized trials and observational studies are evidence that the provision of DHA during earlier stages of brain growth and development can program later brain function. Unfortunately, not only do most published investigations employ only global tests of development, as noted above, but they also stop evaluation well before 4 years of age.

Studies are needed that target outcomes relevant to DHA supplementation and that look at cognition at more appropriate ages. We are currently doing such a study in children from our KUDOS cohort with funding from the National Institute of Child Health and Development to follow a group provided DHA in utero out to 6 years of age with twice yearly developmental assessments. Whether or not future studies will support positive effects on performance in children exposed to higher DHA (or LC-PUFA) at early stages of development (e.g. during gestation and infancy) remains to be seen. Currently, there is enough evidence to suggest this idea is plausible and worthy of study. In the absence of evidence to the contrary, it cannot be concluded that LC-PUFA supplementation does not enhance brain development.

\section{High Variability in Maternal to Fetal Docosahexaenoic Acid Transfer}

At this point, there have been a number of randomized studies that provided DHA to pregnant women with the intent of following development of children born to the pregnancies. It is becoming clear from some of these studies [25] that transfer of DHA from mother to infant is highly variable. Luxwolda et al. [26] have compared groups of women consuming quite different amounts of DHA as part of their cultural food patterns. They found that when maternal DHA status is low, cord DHA levels are higher than in the mother; whereas when maternal DHA status is high, cord blood levels are lower than in the mother. On the other hand, we find that DHA levels in women who have typically low US intakes are sometimes higher and sometimes lower than in cord blood. Moreover, maternal DHA status while significantly positively related to cord blood DHA 
accounts for only about $25 \%$ of the variance in cord blood DHA [25]. Thus, it is clear that there is considerable variability in DHA transfer from mother to fetus. Given that DHA supplementation is provided to women during pregnancy in clinical trials with the intent of increasing DHA transfer to the fetus during a period of rapid brain development, it is obvious that factors that influence variability of transfer could increase the variability in randomized groups and the outcomes of the trials. A concerted effort is needed to determine the predictors of this variability and, we believe, to raise awareness that this variability contributes to considerable overlap in DHA status among study groups. Most randomized trials do not emphasize this variability in publication. Such overlap does not occur in clinical trials that provide formulas with and without DHA to infants, and it may not occur to readers the extent to which it occurs in pregnancy trials. In addition, there is inherent variability in DHA status among women in trials as will be discussed in the next section.

\section{FADS1/2 Single-Nucleotide Polymorphisms and LC-PUFA Requirements}

Friesen and Innis [27] point out the relationship of maternal ARA and DHA with maternal-to-fetal transfer of ARA and DHA and the variability among pregnant women in status. Some of the variability in groups in clinical trials is likely introduced by differences in compliance with supplement use. Another factor that could be responsible within cohorts and that is a known difference among trials is the antecedent DHA status of the woman. Until recently, these differences were believed to be due mainly to differences in maternal dietary intake of DHA. However, a number of papers recently have demonstrated that some single-nucleotide polymorphisms (SNPs) of the fatty acid desaturase genes 1 and 2 (FADS1/2) can influence DHA and ARA status.

Recently, we looked at SNPs of FADS1 rs174553 (the gene that codes for fatty acid $\delta 5$ desaturase) and FADS2 rs174575 (the gene that codes for fatty acid $\delta 6$ desaturase). The minor allele frequency of these two SNPs is 0.28 in our KUDOS cohort with $48 \%$ of subjects carrying one or more copies of a minor allele for each SNP. Women carrying two copies of the rs174553 minor allele had significantly lower DHA status at enrollment, but identical DHA status after supplementation compared to women who carried at least one copy of the major allele. It is well accepted that n-3 LC-PUFA supplementation reduces ARA in membranes. Interestingly, this did not occur except in women carrying one or more copies of the minor alleles for these SNPs, i.e. DHA supplementation resulted in a significant decline in red blood cell membrane ARA in women who carried one or two copies of the minor allele for rs 174553 or two copies of the 
minor allele for rs 174575 [28]. The frequency with which these minor alleles occur in the population suggests that they affect both pre- and postsupplementation n-3 and n-6 LC-PUFA status (and n-3:n-6 fatty acid ratios) in clinical trials [28]. Because these amounts and ratios are linked to functional outcomes, it also suggests that SNPs in FADS1/2 influence requirements for these fatty acids.

At this time, there have been a number of trials to provide DHA during pregnancy in some form. While several of these trials have found effects of maternal DHA status on infant development (much like the observational studies that evaluate infant or child development in relation to maternal DHA status and find positive effects on cognitive outcomes), none of these trials to our knowledge has found a positive effect of randomization to DHA on cognitive outcomes. It seems time to acknowledge that intent-to-treat strategies used in clinical trials to investigate nutrients may have value from a public health perspective but are not helping us determine which pregnancies would benefit from DHA or the best n-3 LC-PUFA or n-3:n-6 LC-PUFA status. There is a need to explore results from these trials to generate new hypotheses.

\section{Conclusions}

In this chapter, we have attempted to highlight directions in studies of LC-PUFA and infant/child development that need additional research based on the current state of knowledge in this area of investigation. Emphasis is placed on the need for employing more targeted measures for assessing early indicators of cognitive function as well as designing studies that are powered to look at ages that may better reflect the effects of early LC-PUFA supplementation on cognitive function. We also emphasize the high variability in DHA status among women and in apparent DHA transfer to the fetus during pregnancy. We do not believe this variability is appreciated by many, and it is a likely contributor to 'no effect' findings in published clinical studies of n-3 LC-PUFA supplementation during pregnancy.

\section{Disclosure Statement}

Our research was supported in part by NIH R01 HD047315 and Mead Johnson Nutrition. 


\section{References}

$\checkmark 1$ Putnam JC, Carlson SE, DeVoe PW, Barness LA: The effect of variations in dietary fatty acids on the fatty acid composition of erythrocyte phosphatidylcholine and phosphatidylethanolamine in human infants. Am J Clin Nutr 1982;36:106-114.

$\checkmark 2$ Clandinin MT, Chappell JE, Leong S, et al: Intrauterine fatty acid accretion rates in human brain: implications for fatty acid requirements. Early Hum Dev 1980;4:121-129.

>3 Clandinin MT, Chappell JE, Leong S, et al: Extrauterine fatty acid accretion in infant brain: implications for fatty acid requirements. Early Hum Dev 1980;4:131-138.

4 Makrides M, Neumann MA, Byard RW, et al: Fatty acid composition of brain, retina, and erythrocytes in breast- and formula-fed infants. Am J Clin Nutr 1994;60:189-194.

5 Farquharson J, Jamieson EC, Abbasi KA, et al: Effect of diet on the fatty acid composition of the major phospholipids of infant cerebral cortex. Arch Dis Child 1995;72:198203.

6 Carlson SE, Werkman SH, Rhodes PG, Tolley EA: Visual-acuity development in healthy preterm infants: effects of marine-oil supplementation. Am J Clin Nutr 1993;58:35-42.

7 Birch EE, Birch DG, Hoffman DR, Uauy R: Dietary essential fatty acid supply and visual acuity development. Invest Ophthalmol Vis Sci 1992;33:3242-3253.

$\checkmark 8$ Werkman SH, Carlson SE: A randomized trial of visual attention of preterm infants fed docosahexaneoic acid until 9 months. Lipids 1996;31:91-97.

$\checkmark 9$ Neuringer M, Connor WE, Van Petten C, Barstad L: Dietary omega-3 fatty acid deficiency and visual loss in infant rhesus monkeys. J Clin Invest 1984;73:272-276.

10 Reisbick S, Neuringer M, Gohl E, et al: Visual attention in infant monkeys: effects of dietary fatty acids and age. Dev Psychol 1997;33: 387-395.

11 Carlson SE, Werkman SH, Rhodes PG, Tolley EA: Visual acuity development in healthy preterm infants: Effect of marine oil supplementation. Am J Clin Nutr 1993;58:35-42.

12 Makrides M, Gibson RA, McPhee AJ, et al: Neurodevelopmental outcomes of preterm infants fed high-dose docosahexaenoic acid: a randomized controlled trial. JAMA 2009;30: $175-182$.
13 Birch EE, Carlson SE, Hoffman DR, et al: The DIAMOND (DHA Intake and Measurement of Neural Development) Study: a double-masked, randomized controlled clinical trial of the maturation of infant visual acuity as a function of the dietary level of docosahexaenoic acid. Am J Clin Nutr 2010;91: 848-859.

14 Makrides M, Gibson RA, McPhee AJ, et al: Effect of DHA supplementation during pregnancy on maternal depression and neurodevelopment of young children: a randomized trial. JAMA 2010;304:1675-1683.

15 Colombo J, Carlson SE: Is the measure the message: the BSID and nutritional interventions. Pediatrics 2012;129:1166-1167.

16 Roze E, Meijer L, Van Braeckel KNJA, et al: Developmental trajectories from birth to school age in healthy term-born children. Pediatrics 2010;126:e1134-e1142.

17 Colombo J: Infant Cognition: Predicting Later Intellectual Functioning. Newbury Park, Sage Publications, 1993.

18 Colombo J, Kannass KN, Shaddy DJ, et al: Maternal DHA and the development of attention in infancy and toddlerhood. Child Dev 2004;75:1254-1267.

19 Kannass KN, Colombo J, Calrson SE: Maternal DHA levels and toddler free-play attention. Dev Neuropsychol 2009;34:159174.

20 Colombo J, Carlson SE, Cheatham CL, et al: Long-chain polyunsaturated fatty acid supplementation in infancy reduces heart rate and positively affects distribution of attention. Pediatr Res 2011;70:406-410.

21 Cheatham CL, Colombo J, Carlson SE: N-3 fatty acids and cognitive and visual acuity development: methodologic and conceptual considerations. Am J Clin Nutr 2006;83: 1458S-1466S.

22 Helland IB, Smith L, Saarem K, et al: Maternal supplementation with very-long-chain n-3 fatty acids during pregnancy and lactation augments children's IQ at 4 years of age. Pediatrics 2003;111:e39-e44.

23 Jensen CL, Voigt RG, Llorente AM, et al: Effects of early maternal docosahexaenoic acid intake on neuropsychological status and visual acuity at five years of age of breast-fed term infants. J Pediatr 2010;157: 900-905. 
24 Colombo J, Carlson SE, Cheatham CL, Shaddy DJ, Kerling EH, Thodosoff JM, Gustafson KM, Brez C: Long-term effects of LCPUFA supplementation on childhood cognitive outcomes. Am J Clin Nutr 2013;98:1-10.

25 Lauritzen L, Carlson SE: Maternal fatty acid status during pregnancy and lactation and relation to newborn and infant status. Matern Child Nutr 2011;7(suppl 2):41-58.

26 Luxwolda MF, Kuipers RS, Sango WS, et al: A maternal erythrocyte DHA content of approximately $6 \mathrm{~g} \%$ is the DHA status at which intrauterine DHA biomagnifications turns into bioattenuation and postnatal infant DHA equilibrium is reached. Eur J Nutr 2012;51:665-675.
27 Friesen RW, Innis SM: Linoleic acid is associated with lower long-chain n-6 and n-3 fatty acids in red blood cell lipids of Canadian pregnant women. Am J Clin Nutr 2010;91: 23-31.

28 Scholtz SA: Influence of FADS1 and FADS2 genotypes on maternal docosahexaenoic acid status and infant cognition; $\mathrm{PhD}$ dissertation, University of Kansas, Medical Nutrition Science, 2012. 